Document downloaded from:

http://hdl.handle.net/10251/141952

This paper must be cited as:

Rincon, J.; Costa, A.; Villarrubia, G.; Julian Inglada, VJ.; Carrascosa Casamayor, C. (01-2). Introducing dynamism in emotional agent societies. Neurocomputing. 272:27-39. https://doi.org/10.1016/j.neucom.2017.03.091

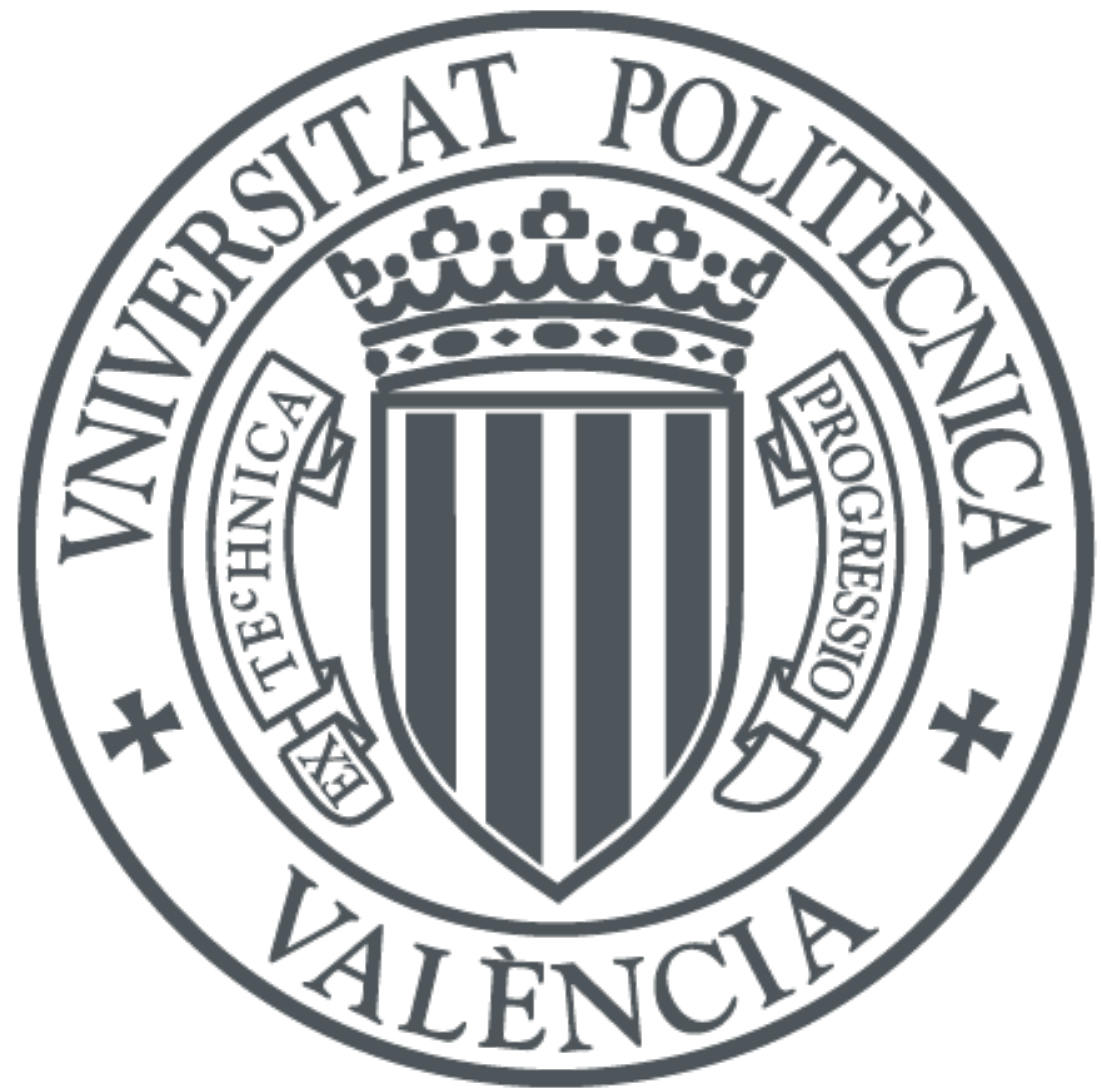

The final publication is available at

https://doi.org/10.1016/j.neucom.2017.03.091

Copyright Elsevier

Additional Information 


\section{Accepted Manuscript}

Introducing Dynamism in Emotional Agent Societies

J.A. Rincon, A. Costa, G. Villarubia, V. Julian, C. Carrascosa

PII:

S0925-2312(17)31115-3

DOI:

10.1016/j.neucom.2017.03.091

Reference: $\quad$ NEUCOM 18616

To appear in:

Neurocomputing

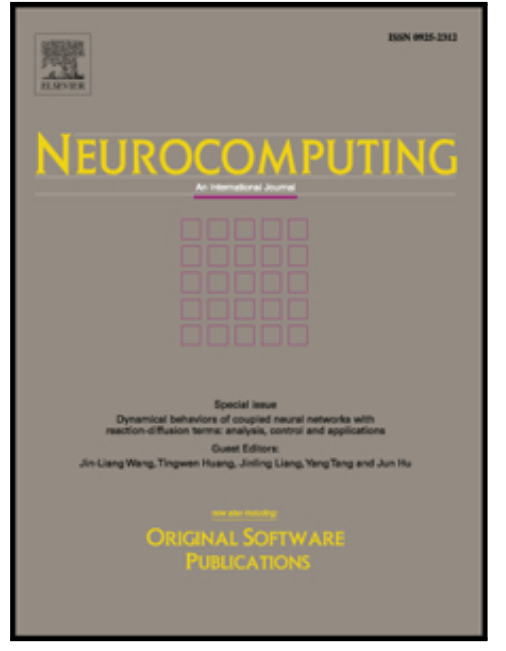

Received date: $\quad 16$ October 2016

Revised date: $\quad 21$ February 2017

Accepted date: $\quad 5$ March 2017

Please cite this article as: J.A. Rincon, A. Costa, G. Villarubia, V. Julian, C. Carrascosa, Introducing Dynamism in Emotional Agent Societies, Neurocomputing (2017), doi: 10.1016/j.neucom.2017.03.091

This is a PDF file of an unedited manuscript that has been accepted for publication. As a service to our customers we are providing this early version of the manuscript. The manuscript will undergo copyediting, typesetting, and review of the resulting proof before it is published in its final form. Please note that during the production process errors may be discovered which could affect the content, and all legal disclaimers that apply to the journal pertain. 


\title{
Introducing Dynamism in Emotional Agent Societies
}

\author{
J. A. Rincon ${ }^{1}$, A. Costa ${ }^{2}$, G. Villarubia ${ }^{3}$, V. Julian ${ }^{1 *}$, C. Carrascosa ${ }^{1}$ \\ ${ }^{1}$ Departamento de Sistemas Informáticos y Computación (DSIC), Universitat Politècnica de València \\ ${ }^{2}$ ISLAB / ALGORITMI, Escola de Engenharia, Universidade do Minho, Braga, Portugal \\ ${ }^{3}$ BISITE, Department of Computer Science, University of Salamanca
}

\section{Abstract}

This paper presents the development of a dynamic emotional model to be employed in agent societies. The proposed model is based on the PAD emotional model and allows the representation of the emotional contagion phenomena of a heterogeneous group of agents that are capable of express emotions. The model is mainly based on three elements: personality, empathy and affinity. These elements allow the characterization of each individual, causing them susceptible to vary in some degree the emotions of other individuals. Additionally, the model allows defining of the social emotion of this group of agents.

Keywords: Multi-agent systems, emotion recognition, neural networks

\section{Introduction}

To attain a person's intentions it is essential to grasp the psychological and the physical aspects. Disregarding one of these aspects may lead to unreliable results [1]. The physiological representation of decisions is a very powerful way to deter5 mine if a person is being honest or not. For instance, a person can be actively lying but the physical response may tell otherwise. Although there are ways to overcome this situation where the person is able to physically control the display of emotions most of the people does not [2].

\footnotetext{
${ }^{*}$ Corresponding author

Email address: vinglada@dsic.upv.es (J. A. Rincon ${ }^{1}$, A. Costa ${ }^{2}$, G. Villarubia ${ }^{3}$, V. Julian ${ }^{1 *}$, C. Carrascosa $a^{1}$ )
} 
Most of the human-computer interaction is solely based on text or clicks, which looses several features present in normal human communication. For instance, the lack of knowledge about one's emotional status constricts the information about the actual disposition (in terms of acceptance/refusal). Furthermore, there are several other factors that influence the emotional status and, therefore, the possible response to a suggestion. Therefore, to enhance the machine decisions it is critical that the complete information about the human/agent in each interaction.

One of the most important influencers of the emotional status in the surrounding environment. Changes in the environment can have an positive or negative outcome (improve the mood or tone down the excitement). From a computational perspective, emotions have been employed as a way to improve social simulation processes which require human interactions, but very little work has been done on representing collective emotions and emotion's dynamicity [3].

Several models have been developed to address emotions and personality so that they are able to recognize and simulate emotions, and they are: OCEAN model [4], OCC emotional models [5], Plutchikś theory [6] and the PAD model [7]. With 25 these models we are able to produce agent systems that are capable of interacting with humans and express emotions. When interacting with other agents they can also perceive the environment. Furthermore, if the agents are equipped with environment sensors (such as cameras[8], speech analysis[9], bio-sensors[10], etc.) they are able to extract the emotional information of humans (although they are 30 still at an early stage).

The issue with the available proposals is that they are static and impervious to change. Normal interactions between the entities (humans or agents) may result in an environment change, like a burst of emotion contagion (here the emotional change of an entity influences other entities). Emotion contagion may be possible when the model takes into account elements such as situation, affinity, empathy, etc.. Until now, only a few works have tried to model the emotional contagion in computational entities: Saunier and Jones[11] modelled the emotional contagion suggesting that each agent is the body and the mind separated; Bosse et al. [12] proposed the spiral model that gives a solution to the emotional propagation by 
distinguishing the different factors that influence the emotional contagion.

Our proposal, which is an extension of a previous work [13], is to introduce a model that is dynamic, which learns from the entities actions and the environment changes. We have based our model on the $P A D$ emotional model with the additional ability of representing the emotional contagion of a heterogeneous group of entities capable of express and/or communicate emotions. Moreover, with this advance it is possible to define the social emotion of a group of agents. To define the model we employ concepts like empathy, affinity and personality of each entity. The aim of this proposal is to attain the complete information about the current emotional status so it can be used on other systems so that they are able to improve their operation.

One of the projects that will benefit from this advance is the iGenda platform $[14,15]$. The iGenda helps managing everyday activities of elderly or disable people. The main features are the scheduling system, the social network and the medical status monitoring. The platform also implements active aging efforts by automatically scheduling activities on the users' free time. One of the issues with this approach is that automatized systems are constructed having one or a few profiles serving as the base options for all the community. Therefore, the people that do not fit on those profiles do not really benefit from the platform features. Our proposal will improve the execution procedures of the iGenda by using virtual actors that have responses similar to human responses. Moreover, in the case of study included in the paper, a robot interacts with the humans calculating the social emotion of the group of people it interacts with and its difference to the goal emotion. This social emotion is calculated from the individual emotions that are obtained from the images of the humans faces captured by the robot. The paper includes the

65 comparison between several methods of machine learning for detecting emotions from face images, being the ANN the method with best results.

To sum up, this paper presents the dynamic model of the Social Emotion concept defined for a group of entities (that may include either humans or agents). This dynamic model may be used to predict the evolution of the group and to identify which individual inside the group can be affected to get the biggest change in the 
Social Emotion. This work is presented in a case study were the emotions from a set of individuals is intended to be detected by capturing their image faces, using an ANN to make this detection.

This paper is structured as follows: section 2 presents the related work and 75 the robotic advances that relate with our concept; section 3 presents the dynamic emotional model and its logical structure; section 4 presents the validation tests of the model presented in section 3; section 5 presents the validation of the model in a real life scenario through the use of a mobile robot; finally, section 6 presents the conclusions and future work.

\section{so 2. Related Work}

The emotional states are defined as the way to express emotion by human beings in a period of time. These emotions are not static and can be propagated through the environment, begin widely used in crowd simulation. It is essential to these applications too have the ability of emulating emotion as they are used to the decision making process. In crowd simulation the most common emotional state is fear, which allows the creation of emergency evacuation simulations [16], [17]. Nevertheless, these simulations try to predict the behavior of humans in distress. These simulations have helped to design buildings, evacuation routes and simulate how the police, firefighters and ambulance may optimally respond to a disaster situation so [18]. However human being have a whole range of emotions that can be propagated to other agents, such as: happiness, sadness and anger, among others. To propagate these emotions the Newtonian Emotion System (NES) [19] was designed for multi-agent systems, establishing the three laws of motion presented by Newton. In the Newton dynamic the aim is the study of movements of objects and the origin of these movements, where each object is represented by a particles system. Each one of these particles have internal properties which makes them different to the other particles properties as the mass, length, with and height, among others this provide to the object a different behaviour when external forces are acting on it. The application of these forces on a particle can changes your direction and velocity 

ronment with the aim of helping people on their daily tasks, track them on their home (and report their health status) and be a sentient companion. Studies show that although current human-robot interactions are far from optimal, future developments would be vital for supporting people [22]. There are several projects

125 directed to assist users and interact with them in an effort to change their mood and influence their emotions. The most active and similar projects are the following.

The Hobbit project [23] is an service robot whose aim is to provide assistance in performing certain tasks. It also interacts with the users, asking them for help to perform tasks that it is unable to do, forcing interaction. It has limited interaction 
The Cosero robot [24] is a humanoid-like attempt that focus more on visual interpretation and grasping. Its aim is to aid to perform home tasks, like cleaning and serving beverages, being able to receive vocal commands or typed messages. It does not posses any type of communicative features, being at its core a service robot.

Lastly, there is the SERGIO robot [25] that is a humanoid robot, that like the previous one, it is a service robot, aiming to perform basic tasks like grasping and object identifying. It is able to communicate using a natural language processor, thus it is capable of basic human communication and keeping a very simple conversation. It is able to receive structured voice command and navigate in an home environment.

The issue with these robots is that they are unable to assimilate the current social and emotional condition of the environment, i.e. they are only capable of performing their tasks whether the user wants it or not. Their strict operation patterns create a distance between them and the humans, being considered as toy by not meeting the user's expectations.

This work tries to overcome this issue by giving an approximation of a dynamic emotional model that allows the representation of the emotional contagion of a heterogeneous group of entities capable of express and/or communicate emotions. The next section explains in detail the proposed model.

\section{Dynamic Emotional Model}

This section proposes a dynamic emotional model based on the PAD emotional model. This model represents the emotional contagion of a heterogeneous group of entities capable of expressing and/or communicating emotions.

Before defining the dynamic emotional model, it is necessary to define the representation of an emotional state of an agent on the PAD model (Pleasure, Arousal and Dominance). The emotion of an agent $a g_{i}$ in an instant $t\left(\vec{E}_{t}\left(a g_{i}\right)\right)$ is defined as a vector in $\mathbb{R}^{3}$, represented by the components that make up the $P A D$ emotional model. The variation of each component allows to modify the emotional state of 
the agent (Equation 1).

$$
\vec{E}_{t}\left(a g_{i}\right)=\left[P_{t}\left(a g_{i}\right), A_{t}\left(a g_{i}\right), D_{t}\left(a g_{i}\right)\right]
$$

This representation in $\mathbb{R}^{3}$ allows us to see emotions as a system of particles. They attract or repel depending on the internal properties of each one of them. These particles have the ability to move around the space because these particles have internal properties like Mass. The mass in a particle is a measure of the amount of matter that has a body, and one of the properties related to it is that it is proportional to the resistance to be attracted by others.

The attraction carried out in the $P A D$ space reflects the emotional contagion between entities. An entity will be more easily suffer from contagion of other emotions according to different factors. The main factor, depending on the own entity is called Empathy. The empathy is a psychological motivator for helping others in distress [26]. The empathy could be defined as the ability to feel what other people feel. The empathy denotes a deep emotional understanding of another's feelings or problems, while sympathy is more general and can apply to small annoyances or setbacks. Our dynamical model uses this psychological concept, allowing agents to have an empathy level. The Empathy Level of an agent $a g_{i}$, denoted $\varepsilon\left(a g_{i}\right)$, represents a value in the range $[0,1]$ indicating the ability of agent $a g_{i}$ to perceive what another agent may feel. In the PAD space, the mass of agent $a g_{i}\left(m\left(a g_{i}\right)\right)$ is defined as the inverse of empathy (Equation 2) as an indicator of the difficult to be attracted by others, that is to be contagied by other emotions as $m\left(a g_{i}\right)$ increases.

$$
m\left(a g_{i}\right)=\frac{1}{\left|\varepsilon\left(a g_{i}\right)\right|}
$$

Another important factor in the emotional contagion is the relationship between the emotion source and the emotion receiver, that is, the Affinity existing between them. It is not the same to perceive the emotions of a close acquaintance than a stranger. The Affinity Level between two agents $a g_{i}$ and $a g_{j}$ at instant $t$ $\left(A f_{t}\left(a g_{i}, a g_{j}\right)\right)$ is a value between $[-1,1]$ that describes the level of affinity between agents $a g_{i}$ and $a g_{j}$, being -1 the value dedicated to sworn enemies, 0 to perfect strangers and 1 to best of friends. The last factor to take into account in 
the emotion dynamics is the physical distance between the emotion source and the emotion receiver $\left(D_{t}\left(a g_{i}, a g_{j}\right)\right.$ to denote the physical distance between entities $a g_{i}$ and $a g_{j}$ at instant $t$ ).

The emotional dynamics described is based on the Newton universal attraction law. Newton's law of universal gravitation states that any two bodies in the Universe attract each other with a force that is directly proportional to the product of their masses and inversely proportional to the square of the distance between them. Based on this theory, we define the force that an agent $a g_{j}$ makes over an agent $a g_{i}$ at instant $t\left(\vec{F}_{t}\left(a g_{i}, a g_{j}\right)\right)$ to attract or repulse it in the PAD space, that is, this force will control the emotion contagion between all the agents. The emotional force is a vector in $\mathbb{R}^{3}$ space. This vector measures the emotional change in the $P A D$ space (Equation 3).

$$
\vec{F}_{t}\left(a g_{i}, a g_{j}\right)=\frac{\varepsilon\left(a g_{i}\right) \cdot A f_{t}\left(a g_{i}, a g_{j}\right)}{2^{D_{t}\left(a g_{i}, a g_{j}\right)}} \cdot\left\|\vec{E}_{t}\left(a g_{i}\right)-\vec{E}_{t}\left(a g_{j}\right)\right\|
$$

$\vec{F}_{t}\left(a g_{i}, a g_{j}\right)$ represents the force véctor, which help us to know if the emotion of the agent $a g_{i}$ is attracted by the agent $a g_{j}, \varepsilon\left(a g_{i}\right)$ represents the emphatic level of entity $a g_{i}$, and $A f_{t}\left(a g_{i}, a g_{j}\right)$ represents the affinity level between $a g_{i}$ and $a g_{j}$ at instant $t . D_{t}\left(a g_{i}, a g_{j}\right)$ is the physical distance between $a g_{i}$ and $a g_{j}$ at instant $t$ and $\vec{E}_{t}\left(a g_{i}\right)$ represents the emotion of the $a g_{i}$ at instant $t$ and $\vec{E}_{t}\left(a g_{j}\right)$ represents the emotion of the $a g$ at instant $t$. According to this, we define the Emotional Attraction Force of agent $a g_{i}$ at instant $t\left(\overrightarrow{E A F}_{t}\left(a g_{i}\right)\right)$ as the combination of all the attraction forces over agent $a g_{i}$ at instant $t$ (Equation 4).

$$
\overrightarrow{E A F}_{t}\left(a g_{i}\right)=\sum_{\forall a g_{j} \neq a g_{i}} \vec{F}_{t}\left(a g_{i}, a g_{j}\right)
$$

To calculate the new emotion of agent $a g_{i}$ at instant $t+1$ and assuming that there is no external stimuli that may change agent $a g_{i}$ emotion out of the rest of entities in the system, it will be calculated according to movement in the $P A D$ space. To get this new emotion it is necessary to use the second law of Newton's or the fundamental principle of dynamics. Based on this law, the $\overrightarrow{E A F}_{t}\left(a g_{i}\right)$ is used to calculate the emotional acceleration of agent $a g_{i}$ at instant $t\left(\vec{a}_{t}\left(a g_{i}\right)\right)$. This acceleration is the emotional variation per time unit of agent $a g_{i}$ emotion (Equation 
$5)$.

$$
\overrightarrow{E A F}_{t}\left(a g_{i}\right)=m\left(a g_{i}\right) \cdot \vec{a}_{t}\left(a g_{i}\right)
$$

Once the emotional acceleration $\vec{a}_{t}\left(a g_{i}\right)$ is calculated, the emotional velocity of entity $a g_{i}$ at instant $t$ can be obtained $\left(\vec{v}_{t}\left(a g_{i}\right)\right)$. This is a measure of the emotional propagation velocity within the $P A D$ space (Equation 6).

$$
\vec{v}_{t}\left(a g_{i}\right)=\vec{a}_{0}\left(a g_{i}\right)+\left(\vec{a}_{t}\left(a g_{i}\right) \cdot t\right)
$$

Finally, it is necessary to calculate the new $P A D$ emotion for entity $a g_{j}$ ) at instant $t+1\left(\vec{E}_{t+1}\left(a g_{j}\right)\right)$ (Equation 7).

$$
\vec{E}_{t+1}\left(a g_{j}\right)=\vec{E}_{t}\left(a g_{j}\right)+\left(\vec{v}_{t}\left(a g_{i}\right) \cdot t\right)
$$

It is important to consider that emotions within the $P A D$ space do not present any opposition by the environment, e.g., there is no friction causing a reduction of speed. There is no inercia affecting the emotions within the PAD space thus, there are no oscillations. This swing up was eliminated by adding this restriction to the model if $\overrightarrow{E A F}_{t}\left(a g_{i}\right)=0$ then $\vec{v}_{t}\left(a g_{i}\right)=0$.

The proposed dynamic model allows us to model and represent the emotional contagion phenomena among different intelligent agents. Nevertheless, these entities typically are not alone in the environment but are part of a group of agents. Our proposal is to model not only how an agent is influenced by other agents but also how the group of agents as a whole can be emotionally affected by its components. To do this, we need to define a social emotional model, which allows to calculate and represent the social emotion of a group of intelligent entities. The aim of this social emotional model is to obtain the social emotion of a group of heterogeneous agents in an specific instant. This model is composed by a triplet that allows us to define the social emotion (SE) [27] for a group of n agents $A g=\left\{a g_{1}, a g_{2}, \ldots, a g_{n}\right\}$ at instant $t$ (Equation 8). 
Where $\overrightarrow{C E}_{t}(A g)$ is a vector in the PAD space, where each one of its components is calculated averaging the $P, A$, and $D$ values, respectively of the $\mathrm{n}$ agents forming the set $A g$ (Equation 9). These averages will enable us to determine where the central emotion $(C E)$ of this group of agents is and to visualize it in the $P A D$ space.

$$
\begin{aligned}
\bar{P}_{t}(A g) & =\frac{\sum_{i=1}^{n} P_{t}\left(a g_{i}\right)}{n}, \bar{A}_{t}(A g)=\frac{\sum_{i=1}^{n} A_{t}\left(a g_{i}\right)}{n}, \bar{D}_{t}(A g)=\frac{\sum_{i=1}^{n} D_{t}\left(a g_{i}\right)}{n}, \\
\overrightarrow{C E}_{t}(A g) & =\left[\bar{P}_{t}(A g), \bar{A}_{t}(A g), \bar{D}_{t}(A g)\right]
\end{aligned}
$$

The $\vec{m}_{t}(A g)$ can indicate if there exist agents having their emotional state far away from the central emotion. The Euclidean distance is used to calculate the maximum distances between the emotion of each agent respect to the $\overrightarrow{C E}$ (Equation $10,11,12,13)$ as follows.

$$
\begin{gathered}
m P_{t}(A g)=\max \left(\sqrt{\left(P_{t}\left(a g_{i}\right)-\bar{P}_{t}(A g)\right)^{2}}\right), \forall a g_{i} \in A g \\
m A_{t}(A g)=\max \left(\sqrt{\left(A_{t}\left(a g_{i}\right)-\bar{A}_{t}(A g)\right)^{2}}\right), \forall a g_{i} \in A g \\
m D_{t}(A g)=\max \left(\sqrt{\left(D_{t}\left(a g_{i}\right)-\bar{D}_{t}(A g)\right)^{2}}\right), \forall a g_{i} \in A g \\
\vec{m}_{t}(A g)=\left[m P_{t}(A g), m A_{t}(A g), m D_{t}(A g)\right]
\end{gathered}
$$

The $\vec{\sigma}(A g)$ or standard deviation (SD) allows the calculation of the level of emotional dispersion of this group of agents around the central emotion $\overrightarrow{C E}(A g)$ for each component of the $P A D$ (Equation 14).

$$
\begin{gathered}
\sigma P_{t}(A g)=\sqrt{\frac{\sum_{i=1}^{n}\left(P_{t}\left(a g_{i}\right)-\bar{P}_{t}(A g)\right)^{2}}{n}}, \forall a g_{i} \in A g \\
\sigma A_{t}(A g)=\sqrt{\frac{\sum_{i=1}^{n}\left(A_{t}\left(a g_{i}\right)-\bar{A}_{t}(A g)\right)^{2}}{n}}, \forall a g_{i} \in A g \\
\sigma D_{t}(A g)=\sqrt{\frac{\sum_{i=1}^{n}\left(D_{t}\left(a g_{i}\right)-\bar{D}_{t}(A g)\right)^{2}}{n}}, \forall a g_{i} \in A g
\end{gathered}
$$


The result of each of the above equations can be represented as a vector (Equation 15), which allow to determine the level of emotional dispersion.

$$
\vec{\sigma}_{t}(A g)=\left[\sigma P_{t}(A g), \sigma A_{t}(A g), \sigma D_{t}(A g)\right]
$$

From this definition, it can be deduced that:

1. if $\vec{\sigma}_{t}(A g)>>[0,0,0]$, the group has a high emotional dispersion, i.e. the members of the group have different emotional states.

2. if $\vec{\sigma}_{t}(A g) \cong[\mathbf{0 , 0}, \mathbf{0}]$, the group has a low emotional dispersion, this means that individuals have similar emotional states.

This model takes into account that at some stage you may have two or more agent groups and each group has its own social emotion or have a single group which wants to move to a target emotion. This will allow to measure the emotional distance between the current social emotional group and a possible emotional target. This approach can be used as a feedback in the decision making process in order to take actions to try to move the social emotion to a particular area of the $P A D$ space or to allow that the emotional state of a group of agents can be approached or moved away from other groups of agents (Equation 16).

$$
\Delta_{S E}: S E_{t}\left(A g^{i}\right), S E_{t^{\prime}}\left(A g^{j}\right) \rightarrow[0,1]
$$

According to this profile, Equation 17 shows how we calculate this emotional variation. The equation calculates three distances corresponding to the three components of the $S E$.

$$
\begin{gathered}
\Delta_{S E}\left(S E_{t}\left(A g^{i}\right), S E_{t^{\prime}}\left(A g^{j}\right)\right)=\frac{1}{2}\left(\omega_{c} \Delta\left(\overrightarrow{C E}_{t}\left(A g^{i}\right), \overrightarrow{C E}_{t^{\prime}}\left(A g^{j}\right)\right)\right. \\
\left.+\omega_{d} \Delta\left(\vec{m}_{t}\left(A g^{i}\right), \vec{m}_{t^{\prime}}\left(A g^{j}\right)\right)+\omega_{v} \Delta\left(\vec{\sigma}_{t}\left(A g^{i}\right), \vec{\sigma}_{t^{\prime}}\left(A g^{j}\right)\right)\right) \\
\text { where } \omega_{c}+\omega_{d}+\omega_{v}=1 ; \quad \omega_{c}, \omega_{d}, \omega_{v} \in[0,1]
\end{gathered}
$$

and $\Delta$ calculates the distance between two vectors. As every dimension of the $P A D$ space is bounded between $[-1,1]$, each $\Delta$ will give values between $[0,2]$. Therefore, $\Delta_{S E}$ will have a range between $[0,1]$. Calculating the distance among 
social emotions allows the study of the behaviour of emotional-based agents, either minimizing or maximizing the $\Delta_{S E}\left(S E_{t}\left(A g^{i}\right), S E_{t^{\prime}}\left(A g^{j}\right)\right)$ function. This way, it can be extrapolated the knowledge about if an agent group approaches or moves away from a specific emotional state. To achieve this, it is necessary to modify through stimuli the individual emotions of each agent and therefore changing the social emotion.

Using this model is possible to determine the emotional distance among different groups of agents or between the same group in different instants of time. This will allow us to measure the emotional distance between the current social emotional group and a possible emotional target. Moreover, the combination of the presented models allows us to model and represent the emotional contagion of a heterogeneous group of agents and observe how it influences the social emotion of that group of agents.

\section{Validation tests}

Different tests have been done in order to validate the proposed model. Concretely, a simulation prototype was implemented in Python (using a jupyter ${ }^{1}$ notebook with numpy and matplotlib libraries). The simulation experiments were conducted to evaluate different aspects and to try to show the correct behavior of the proposed model. Visualization of results has been done using three different kind of images:

- PAD space representation: a 3D representation of the emotional states in the PAD space. In each graphic, current emotional states of each agent and the social emotion of the existing groups are represented.

- Physical space position representation: a 2D representation of the different agents, similar to a graph where each agent is a node situated in its physical coordinates $(\mathrm{x}, \mathrm{y})$. The size of the agent is inversely proportional to its empathy and if there is any affinity between agents, it will be represented by a link

\footnotetext{
${ }^{1}$ http://jupyter.org
} 
joining them. Finally, a sequence of colors (see Figure 1) is defined as a way for representing the current emotion of each agent.

- Social emotional evolution: a 2D representation of the evolution of the different values composing the Social Emotion $\left(S E_{t}(A g)\right)$ :

- $\overrightarrow{C E}_{t}(A g)=\left[\bar{P}_{t}(A g), \bar{A}_{t}(A g), \bar{D}_{t}(A g)\right]$, represented in the figure as $C E P$, $C E A$ and $C E D$, respectively.

- $\vec{m}_{t}(A g)=\left[m P_{t}(A g), m A_{t}(A g), m D_{t}(A g)\right]$, represented in the figure as maxDistP, maxDistA and maxDistD, respectively.

- $\vec{\sigma}_{t}(A g)=\left[\sigma P_{t}(A g), \sigma A_{t}(A g), \sigma D_{t}(A g)\right]$, represented in the figure as $s t d P, s t d A$ and $s t d D$, respectively.

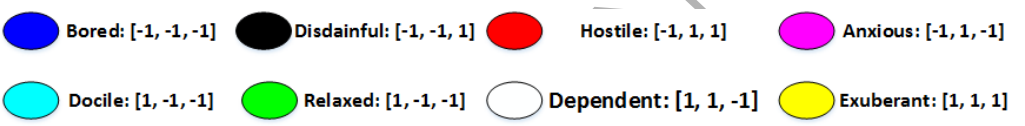

Figure 1: Color representation for the different emotions

The experiments have been grouped into three situations changing the characteristics of the agents' groups. Moreover, each experiment includes different cases changing the affinity and empathy levels of the agents and also the physical distance among agents. The different proposed experiments are listed in Table 1.

\subsection{First Experiment}

The first experiment tried to evaluate how a group of heterogeneous agents evolve in the emotional space according to the dynamic model. To do this, we implemented a set of 10 agents with a randomized initial emotional state. In order to evaluate the emotional behavior in the agent group, different situations have been defined changing the empathy and affinity values of each agent. Moreover the maximum distance of 20 meters. For reasons of brevity only two of the combinations are described. 


\begin{tabular}{|c|c|c|c|c|c|c|}
\hline \multirow{2}{*}{ Experiment } & \multirow{2}{*}{\multicolumn{2}{|c|}{ \# agents }} & \multirow{2}{*}{ Empathy } & \multirow{2}{*}{ Affinity } & \multicolumn{2}{|c|}{ Physical distance } \\
\hline & & & & & Case 1 & Case 2 \\
\hline $1 \mathrm{st}$ & 1 group of 10 agents & $\begin{array}{l}\text { a) } \\
\text { b) } \\
\text { c) } \\
\text { d) }\end{array}$ & $\begin{array}{l}0 \\
0 \\
1 \\
1\end{array}$ & $\begin{array}{l}0 \\
1 \\
0 \\
1\end{array}$ & $\begin{array}{c}\text { All agents } \\
\text { have distance } \\
0\end{array}$ & $\begin{array}{l}\text { All agents have } \\
\text { random distances } \\
\text { between } 0 \text { and } 20\end{array}$ \\
\hline 2nd & $\begin{array}{c}1 \text { group of } 10 \text { agents } \\
\text { (one agent with Empathy } \\
\text { and Affinity }=0 \text { ) }\end{array}$ & $\begin{array}{l}\text { a) } \\
\text { b) } \\
\text { c) } \\
\text { d) }\end{array}$ & $\begin{array}{l}0 \\
0 \\
1 \\
1\end{array}$ & $\begin{array}{l}0 \\
1 \\
0 \\
1\end{array}$ & $\begin{array}{c}\text { All agents } \\
\text { have distance } \\
0\end{array}$ & $\begin{array}{l}\text { All agents have } \\
\text { random distances } \\
\text { between } 0 \text { and } 20\end{array}$ \\
\hline 3rd & $\begin{array}{l}1 \text { group of } 5 \text { agents } \\
\text { and } 1 \text { group of } 10 \text { agents }\end{array}$ & $\begin{array}{l}\text { a) } \\
\text { b) } \\
\text { c) } \\
\text { d) }\end{array}$ & $\begin{array}{l}0 \\
0 \\
1 \\
1\end{array}$ & $\begin{array}{l}0 \\
1 \\
0 \\
1\end{array}$ & $\begin{array}{l}\text { All agents } \\
\text { have distance } \\
0\end{array}$ & $\begin{array}{l}\text { All agents have } \\
\text { random distances } \\
\text { between } 0 \text { and } 20\end{array}$ \\
\hline
\end{tabular}

Table 1: Summary of proposed experiments

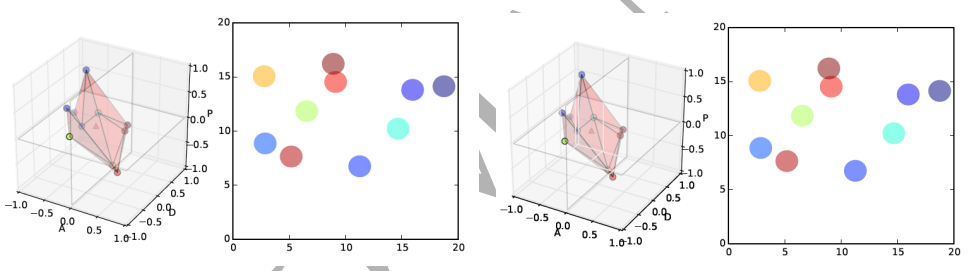

(a) Initial

(b) Final

Figure 2: One group of 10 agents (with Empathy $=0$, Affinity $=0$ and distance between agents $>0$ )

First one is the corresponding to all the empathies and affinities between agents to 0 , that is, a set of agents that has not any relationship between them and that are not moved by the emotions they feel around them. In this situation, the model works as expected, as the agents do not change their emotions. Figure 2 shows one execution of this first situation of this example by a PAD space representation and a Physical space position representation for the initial and final stages of the execution.

Alternatively, Figure 3 represents a situation where agents have a maximum value of the empathy and affinity levels. As we can see, the initial stage for the PAD values of the agents is the same of the previous situation (as can be observed in the 


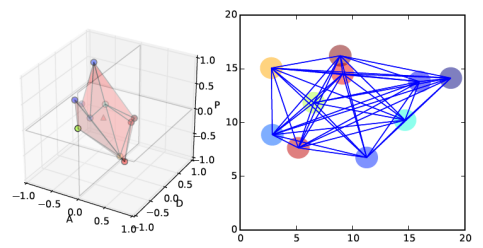

(a) Initial

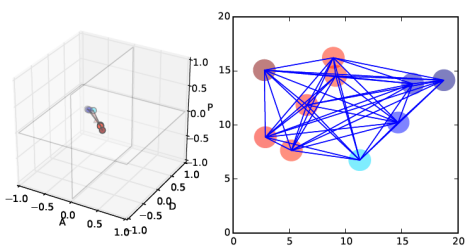

(b) Final

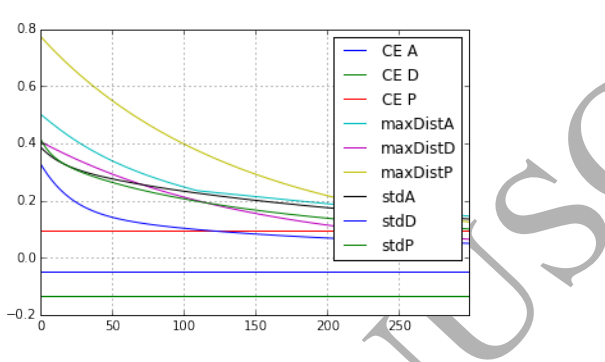

(c) Social emotional evolution

Figure 3: One group of 10 agents (with Empathy=1, Affinity=1 and all the agents with distance $>0$ )

corresponding PAD space representation). As this situation has different affinities and empathies, there exists links connecting agents in the Physical space position representation. This situation represents a group of agents that can be considered good friends and very sensitive to their friends emotions. As they are close enough (in a range of $[0,20]$ meters), their emotions are contaged tending to collapse in the PAD space (as is observed in the Figure $4 \mathrm{~b}-$ left). This evolution can be observed, at individual level, in the evolution of the PAD space representation, and in the evolution of the colors of the agents in the PAD space representation and in the Physical space position representation. On the other hand, Figure $4 \mathrm{c}$ shows how fast is the convergence of the social emotional values during the experiment. The relevance of these experiments is the validation that all the situations have the expected behavior according to the proposed model.

340 4.2. Second Experiment

The second experiment is trying to observe how the emotional state of the group is disturbed by an odd agent without empathy and affinity with any agent. Scenarios 
proposed in this experiment are affected in the emotional states of the group due to the emotional response generated by the odd agent. As an example we can see the scenario proposed in Figure 5a where all the agents of the group have the maximum value of the empathy and affinity levels except the odd agent (an initial situation similar to the one used in the Figure 4a). As we can see, the final situation shows a non perfect grouping of all the agents due to the distorsion caused by the odd agent. This can be observed too in the temporal evolution of the social emotional values, if compared with Figure 3.

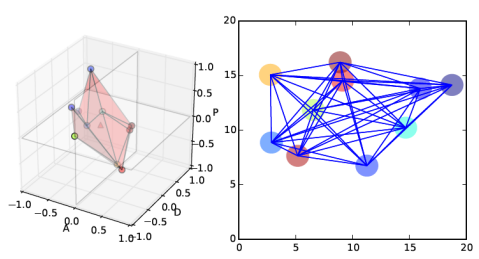

(a) Initial

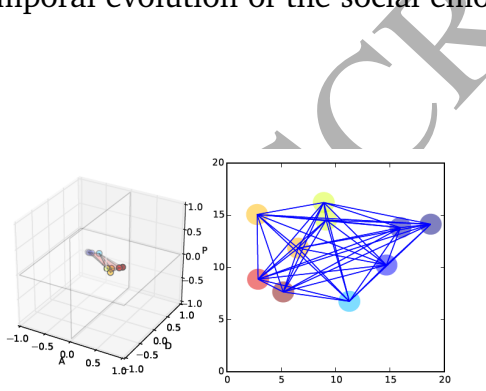

(b) Final

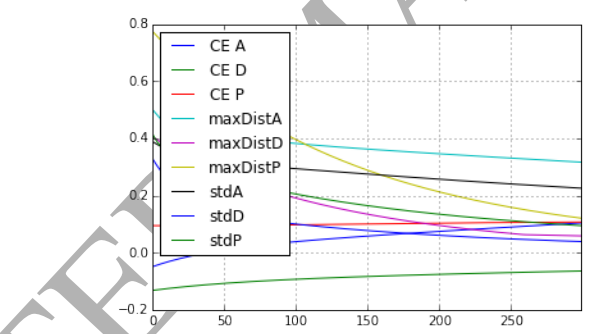

(c) Social emotional evolution

Figure 4: One group of 10 agents with an odd agent (with Empathy=1, Affinity=1 and all the agents with distance $>0$ )

\subsection{Third Experiment}

Finally, the third experiment was centered in analyzing how two disimilar groups of agents change their emotional states following the proposed model.

Figure 5 represents a scenario where there exists one group of ten agents and another group of five agents with the maximum level of empathy and affinity inside the group and the minimum distance between them. In this case, agents of each 


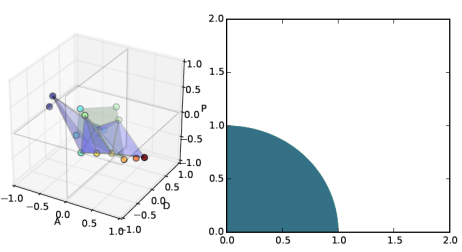

(a) Initial

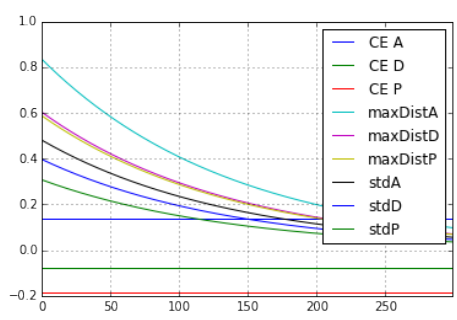

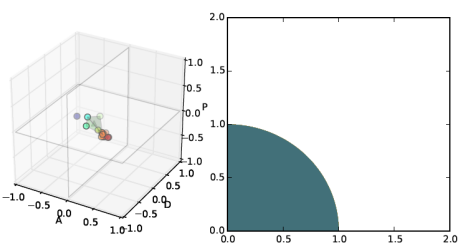

(b) Final

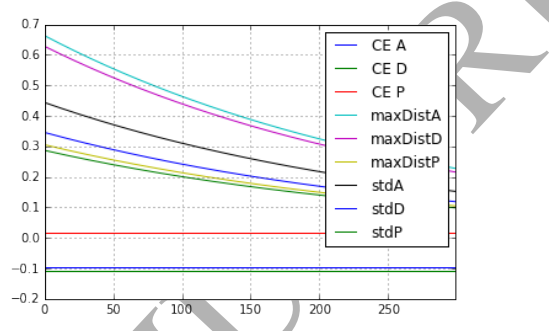

(c) Social emotional evolution of 5 agents(d) Social emotional evolution of 10 agents

Figure 5: One group of 10 agents and another group of 5 agents (with Empathy=1, Affinity=1 and all the agents with distance $=0$ )

group are close to each other as can be expected. Regarding the temporal evolution, it is more evident in the case of the smallest group, where the social emotional parameters are more homogeneous than in the largest group.

To prove the applicability of this model we propose the implementation of it in a mobile robot and proceeded to test different scenarios either in simulated or real environments. The architecture and experiment results are presented in the next section.

\section{A robot guided by emotions.}

The model implementation and the proof of concept was done through the use of a real environment, where there is a NAO robot ${ }^{2}$ in charge of interacting with humans in a room. The main goal of this development is the automatic recognition

\footnotetext{
${ }^{2}$ https://www.aldebaran.com/en
} 
of the emotional states of a group of individuals in order to enhance the wellbeing of these individuals. To achieve this, the robot moves around the room and tries to interact with any detected person. The robot calculates the emotional states of the identified individuals' group and, according to the proposed model, estimates possible emotional contagions among individuals. In order to make this process it uses different tools to communicate with its environment and to obtain the information that surrounds it:

- Speech recognition, the robot communicates with people to try to change their emotional states. Moreover, if the robot does not know the person, it estimates his personality using a dialogue game that follows the OCEAN test [4].

- Movement, the robot is continually moving around the room trying to interact and stimulate any individual presented in the room.

- Image processing, it is used to detect the emotional state of people around the room. To detect the emotional states, the robot employs a machine learning model explained in the next section.

Figure 6 shows a simulated environment of the proposed application where the 385 NAO robot interacts with a group of three individuals.
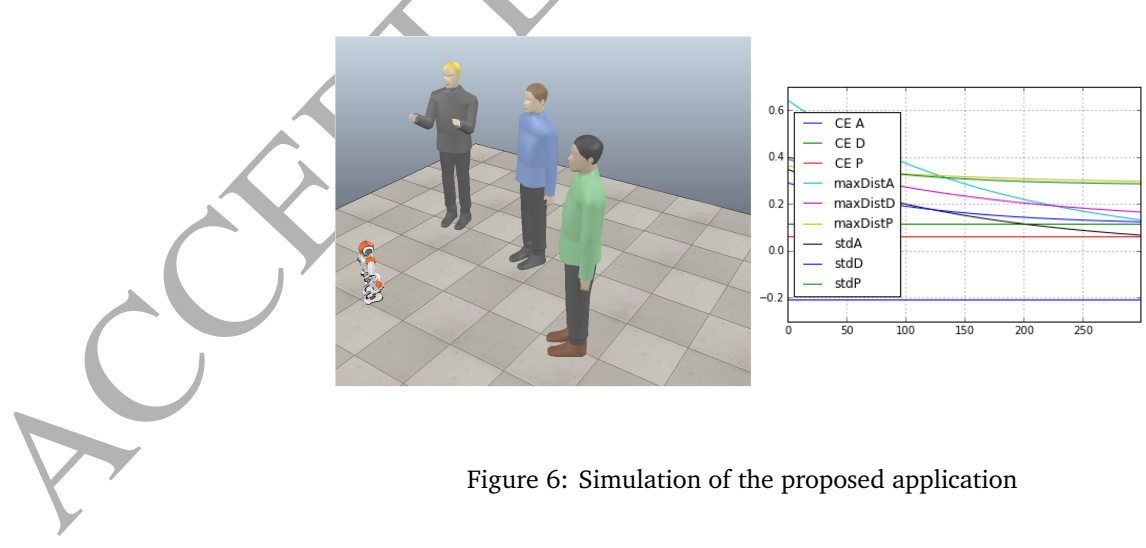

Figure 6: Simulation of the proposed application

With this information, the robot tries to stimulate people in the room. These stimulation actions are performed when the robot detects emotional changes that 
lead the social emotion away from a target emotion (happiness, usually). This continuous sensorization of the environment enables the estimation of the emotional

\subsection{Emotion identification}

In this section we will focus on the design and implementation of the emotion identification of each person which is in the room.

The emotion state detection represents the knowledge about human feelings perceived by the machine (Robot, Mobile Phones, etc). The detection of this emotion is done through a different algorithm, that gives machines this skill and allows them to recognize and classify emotional states. To recognize the emotional state, we can find different algorithms and techniques that extract facial information. Among them, we highlight the Histogram Oriented Gradient (HOG) [29], and the Face Landmark Estimation [30]. The first one is used to encode these facial components and concatenate them into a single feature vector; the other is the technique we have used, and it extracts a list of points as shown in the Figure 7. These points represent the most important characteristics of our faces represented in a 2D plane. 
Using this information, it is possible to create a set of feature vectors that can be used to train the machine learning models $(M L)$. To determine the best feature vector for the classification of emotions, three experiments were performed. The experiments used two databases, that had the same kind of emotions. The emotions contained in these databases are: Afraid, Angry, Disgusted, Happy, Neutral, Sad and Surprised. The first one, called Karolinska Directed Emotional Faces database (KDEF) [31], is used to train our $M L$ and it is composed of 980 images and the second database, The Radboud Faces Database (RaFD) [32], is used to do the test and it is composed of 536 images.

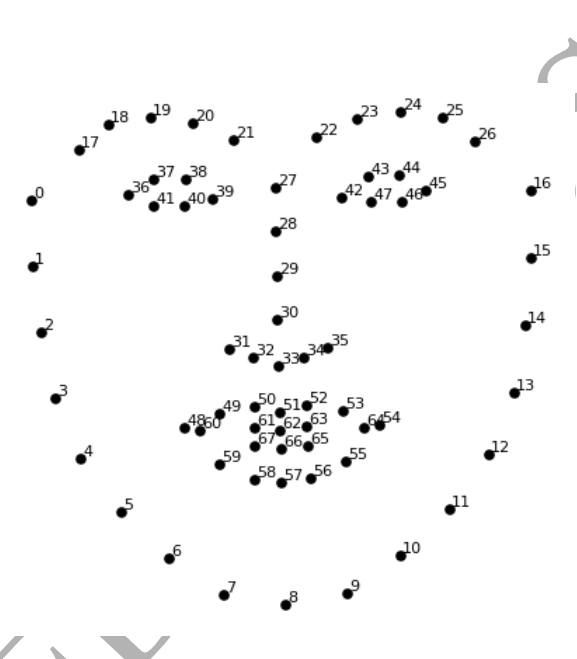

Figure 7: List of extracted points, using Face Landmark Estimation

Table 2 shows the database distribution and the percentage used to train and to test. These databases are composed by different faces that represent human emotions. To do a good classification, it is necessary to detect a series of face characteristics. These points are showed in Figure 8. Based on these points, we calculate the Euclidean distance between each of them. In our experiments, seventeen distances were used, that represent the input used to train the $M L$.

We use agents to represent the human emotions, being able to fully interact with humans. To be able to do this, first, the agent has to possess the ability of classify each emotion, thus possessing a knowledge base with information of a classifier. 


\begin{tabular}{|l|c|c|c|}
\hline Database Name & Total Images & Train & Test \\
\hline KDEF & 980 & 80 & 20 \\
\hline RafD & 469 & 80 & 20 \\
\hline KDEF and RafD & 1449 & 80 & 20 \\
\hline
\end{tabular}

Table 2: Database distribution.

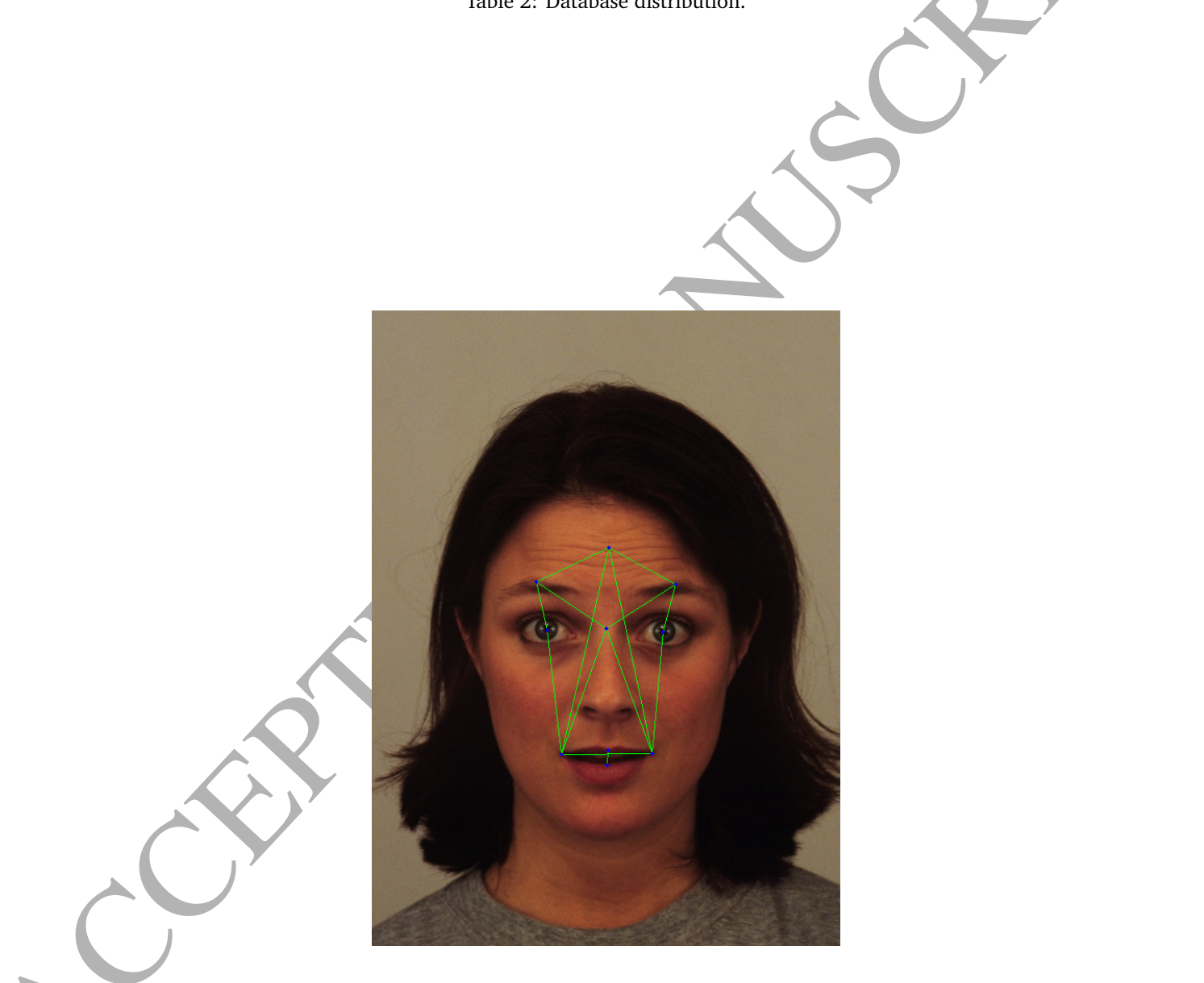

Figure 8: Points used to calculate the euclidean distance. 


\begin{tabular}{|l|l|}
\hline Name & Description \\
\hline C & Penalty parameter C of the error term. \\
\hline Gamma & Radius of influence of samples selected by the model as support vectors. \\
\hline Max Depth & The maximum depth of the tree. \\
\hline n Estimators & The number of trees in the forest. \\
\hline Max Features & The number of features to consider when looking for the best split. \\
\hline Penalty & Used to specify the norm used in the penalization \\
\hline Degree & Degree of the polynomial kernel function. \\
\hline
\end{tabular}

Table 3: Description of values used in the machine learning models.

\begin{tabular}{|l|c|c|c|c|c|c|c|}
\hline Name Classifier & C & Gamma & Max Depth & n Estimators & Max Features & Penalty & Degree \\
\hline SVC Linear Kernel & 1.0 & 0.1 & - & - & - & - & - \\
\hline LinearSVC & 1.0 & - & - & - & - & - & - \\
\hline SVC (RBF kernel) & 1.0 & 0.1 & - & - & - & - & - \\
\hline SVC Polynomial (degree 3) & 1.0 & 0.1 & - & - & - & - & 3 \\
\hline SVC Kernel Sigmoid & 1.0 & 0.1 & - & & - & - & - \\
\hline Logistic Regression & - & - & - & - & - & 12 & - \\
\hline Logistic Regression 1 My caption & - & - & - & - & - & 12 & \\
\hline Gaussian NB & - & - & - & - & - & - & - \\
\hline Random Forest Classifier & - & - & 14 & 10 & 7 & - & - \\
\hline
\end{tabular}

Table 4: Machine Learning Models and their configuration values.

To properly choose from the available methods (neural networks [33], support vector machines [34], etc.), we have performed a comparison between them and chose the one with better results.

Table 3 describes the different configuration values used in the machine learning models. Table 4 shows the different values of the configuration parameters used in the machine learning models.

The result of the classification can be seen in Table 5. The first column corresponds to the KDEF database, the second column corresponds to the RaFD database and the last one are the two joined databases. In each one of these experiments, the relation between the amount of samples used to train and to test was $80 \%$ to train and 20\% to test. Other experiments were performed using Artificial Neural Networks (ANN), with the same database and partition ( $80 \%$ for training, $20 \%$ for test) as the other ones. The topology used in our ANN was defined as: input layer 


\begin{tabular}{|l|c|c|c|}
\hline Classifier Name & KDEF & RafD & KDEF and RafD \\
\hline SVC Linear Kernel & 63 & 29 & 95 \\
\hline LinearSVC & 57 & 30 & 79 \\
\hline SVC (RBF kernel) & 57 & 30 & 79 \\
\hline SVC Polynomial & 57 & 29 & 79 \\
\hline SVC Kernel Sigmoid & 59 & 30 & 92 \\
\hline Logistic Regression & 60 & 26 & 86 \\
\hline Logistic Regression 1 & 65 & 34 & 96 \\
\hline Random Forest Classifier & 71 & 35 & 98 \\
\hline Background Propagation & $\mathbf{7 5 . 2}$ & $\mathbf{6 5 . 5}$ & $\mathbf{9 8 . 5}$ \\
\hline
\end{tabular}

Table 5: Results of the classifiers for each of the databases

seventeen neurons, in the middle layer one hundred and output layer seven neurons.

Using this configuration it was achieved $97 \%$ of correct human emotions classification. It is very important to take into account that all the training process has been made offline, and once the best configuration has been obtained, it is embedded in the agent (in this case, the robot).

Table 5 shows that the best results were obtained joining the two databases.

Nevertheless, it is possible to use all the classifiers presented combined as a group of experts. This group of experts elassify the emotions and count the number of times each emotion appears. Using this information the agents can determine which is the recognised emotion and the emotional state is added to its knowledge base. Once this emotion has been added, the agents may carry out the necessary actions to interact with the person.

\subsection{Emotion dynamics experimentation}

To validate our dynamic emotional model we have defined several experiments to compare the results obtained in simulation with reality. To make this comparison, it is necessary to have an initial information about the participants. This information will be used as an input parameter in the simulation. To obtain this information, the participant answers a series of questions from a personality test (the OCEAN, as previously mentioned). Using this test, it is possible to determine the level of empathy of each participant (using some studies [35] that associates Agreeableness component to empathy) and a list of the affinity levels between the participants. The name 


\begin{tabular}{|l|l|l|l|l|}
\hline & Agent 0 & Agent 1 & Agent 2 & Agent 3 \\
\hline Empathy & 0.3 & 0.9 & 0.6 & 0.5 \\
\hline
\end{tabular}

Table 6: Group 1: Empathy levels of each agent.

\begin{tabular}{|c|c|c|c|c|}
\hline & Agent 0 & Agent 1 & Agent 2 & Agent 3 \\
\hline Agent 0 & 0.0 & 0.8 & 0.75 & 0.95 \\
\hline Agent 1 & 0.79 & 0.0 & 0.89 & 0.85 \\
\hline Agent 2 & 0.86 & 0.76 & 0.0 & 0.79 \\
\hline Agent 3 & 0.8 & 0.8 & 0.95 & 0.0 \\
\hline
\end{tabular}

Table 7: Group 1: Level of affinity between agents. ship levelbetween them is represented in the affinity matrix shown in Table 7.

In the first experiment, the initial emotion of each participant was detected to be as showed in the Table 8 . In this table, the different agents' emotions and their corresponding values in $P A D$ can be seen.

485 Figure 9-b shows the representation of the social emotion dynamics according to the real world execution. These emotions were detected by using the machine learning algorithm presented in previous sections. The delay between captures was of 2 minutes and this process is repeated for one hour. Each emotion detected with our algorithm was transformed in PAD values. 


\begin{tabular}{|c|c|c|c|c|}
\hline & P & A & D & Emotion \\
\hline Agent 0 & 0.63 & 0.40 & 0.29 & Happy \\
\hline Agent 1 & 0.63 & 0.40 & 0.28 & Happy \\
\hline Agent 2 & 0.41 & 0.55 & 0.19 & Surprise \\
\hline Agent 3 & 0.63 & 0.40 & 0.29 & Happy \\
\hline
\end{tabular}

Table 8: Group 1 - Experiment 1: Initial Emotion and PAD values of each agent.

The aim of this simulation is to check how the emotional dynamics work in a group. The Figure 9- $a$ shows the emotional dynamics for 30 seconds. It can be seen how the agents attract each other. This can be observed in the tendency to zero of the dispersion (standard deviation) and the max distance values. This tendency indicates that the agents are grouped around a central emotion represented by $C E$ P, CE A, CE D, that in this case is happiness.

If we compare the two graphics we can observe that the emotional dynamics in the two examples have had the same behaviour.

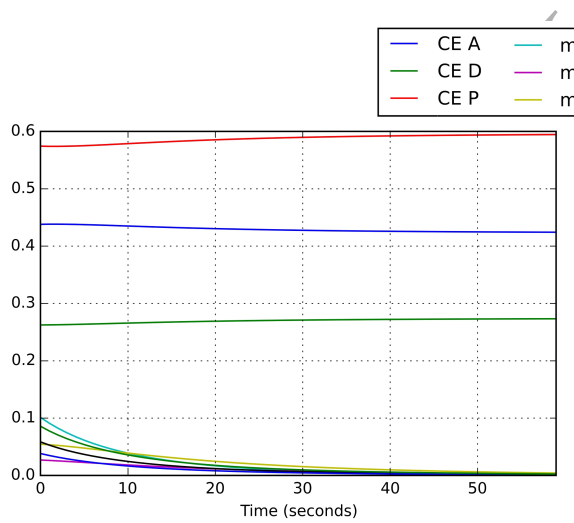

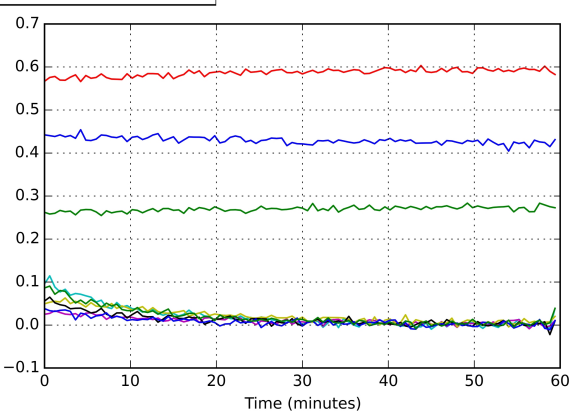

b

Figure 9: Group 1 - Experiment 1: High Affinity levels and positive emotions.

The second experiment with this group have the initial emotions situation perceived that can be seen in Table 9. This second experiment deals about negative 500 emotions' evolution.

In a similar way as in the first experiment, this one was first executed in the real 


\begin{tabular}{|c|c|c|c|c|}
\hline & $\mathrm{P}$ & $\mathrm{A}$ & $\mathrm{D}$ & Emotion \\
\hline Agent 0 & -0.59 & 0.08 & 0.47 & Angry \\
\hline Agent 1 & -0.59 & -0.01 & 0.40 & Disgusted \\
\hline Agent 2 & -0.08 & 0.18 & -0.39 & Fearful \\
\hline Agent 3 & -0.28 & -0.12 & -0.37 & Sad \\
\hline
\end{tabular}

Table 9: Group 1 - Experiment 2: Emotion and PAD values of each agent.

world, and the emotional evolution perceived in Figure10-b.

In the Figure 10- $a$ we can see the simulation evolution of the emotional dynamics using as initial values of the agents' emotions the ones in Table 9.

As this group of participants possess a big empathy and affinity level between them, in both experiments, the group tends to be emotionally cohesive. This can be seen in the figures as the dispersion (standard deviation) and the maximum distance tend to zero. In this second experiment, the central emotion is around Disgusted emotion.

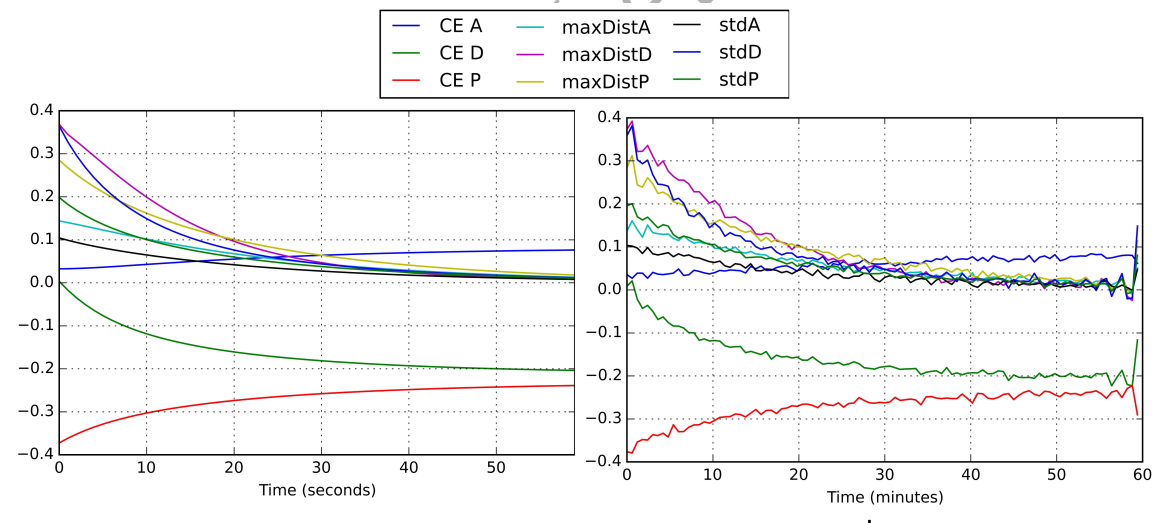

Figure 10: Group 1 - Experiment 2: High Affinity levels and negative emotions.

$510 \quad$ 5.2.2. Group 2

This second group of participants is formed by a group of 4 people that are practically unknown between them (this can be seen in Table 11 where the affinity 


\begin{tabular}{|l|l|l|l|l|}
\hline & Agent 0 & Agent 1 & Agent 2 & Agent 3 \\
\hline Emphaty & 0.1 & 0.5 & 0.8 & 0.95 \\
\hline
\end{tabular}

Table 10: Group 2: Empathy levels to each agent.

\begin{tabular}{|c|c|c|c|c|}
\hline & Agent 0 & Agent 1 & Agent 2 & Agent 3 \\
\hline Agent 0 & 0.0 & 0.1 & 0.01 & 0.05 \\
\hline Agent 1 & 0.09 & 0.0 & 0.04 & 0.06 \\
\hline Agent 2 & 0.01 & 0.06 & 0.0 & 0.07 \\
\hline Agent 3 & 0.08 & 0.02 & 0.03 & 0.0 \\
\hline
\end{tabular}

Table 11: Group 2: Level of affinity between agents.

matrix of this group is shown). Table 10 shows the empathies of such people.

The first experiment with this second group have the initial emotions perceived that are seen in Table 12.

Figure 11- $b$ shows the social emotion dynamics in the real world execution of this firs experiment with the second group of participants. The data acquisition was similar to the one made in the first group experiments.

Figure 11-a shows the results of the social emotion dynamics' simulation using as initial emotion values the ones perceived in the real scenario.

In this first experiment the group maintains its emotional state very close to happiness. In the same way, the dispersion values and maximum distances are very close to zero, indicating that the group is responding positively. This behaviour is not produced by the affinity levels, since in this experiment they are very low. This behaviour is caused by the individuals empathy levels, as two of them have high empathy values.

For the second experiment of the second group of participants, the initial emo-

\begin{tabular}{|c|c|c|c|c|}
\hline & P & A & D & Emotion \\
\hline Agent 0 & 0.63 & 0.40 & 0.29 & Happy \\
\hline Agent 1 & 0.63 & 0.40 & 0.28 & Happy \\
\hline Agent 2 & 0.41 & 0.55 & 0.19 & Surprise \\
\hline Agent 3 & 0.63 & 0.40 & 0.29 & Happy \\
\hline
\end{tabular}

Table 12: Experiment 2A: Initial Emotion and PAD values of each agent. 


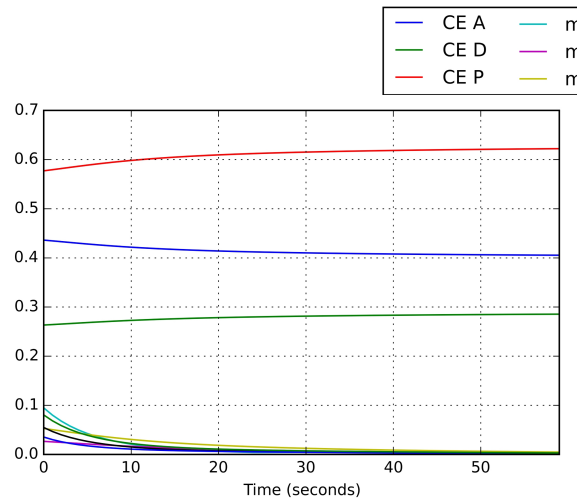

$$
\begin{aligned}
& \text { maxDistA - stdA } \\
& \begin{array}{l}
\text { axDistD }- \text { stdD } \\
\text { maxDistP }
\end{array}
\end{aligned}
$$

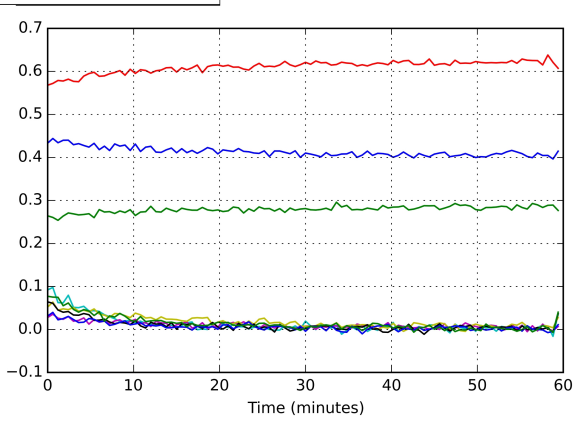

Figure 11: Group 2 - Experiment 1: Low Affinity levels and positive emotions.

\begin{tabular}{|c|c|c|c|c|}
\hline & $\mathrm{P}$ & $\mathrm{A}$ & $\mathrm{D}$ & Emotion \\
\hline Agent 0 & -0.08 & 0.18 & -0.39 & Fear \\
\hline Agent 1 & -0.59 & 0.08 & 0.47 & Angry \\
\hline Agent 2 & -0.59 & -0.01 & 0.40 & Disgusted \\
\hline Agent 3 & -0.28 & -0.12 & -0.37 & Sad \\
\hline
\end{tabular}

Table 13: Group 2 - Experiment 2: Initial Emotion and PAD values of each agent.

tions perceived can be seen in the Table 13.

The results of this experiment in the real world can be seen in the Figure12- $b$, and the simulation of the social emotion dynamics in the Figure12- $a$.

In this second experiment of the second group, we can see that the emotional dispersion and the maximum distance are high. This happens because the levels of affinity between the agents are low. This behaviour occurs both in simulation and in the real world.

535 In the end, we may observe that the social emotion dynamics model seems to work quite properly, as it is able to predict the emotion dynamics, using the affinity levels, empathies and the emotions detected by the cameras. This could be used to anticipate the different actions that can be done so that the group may move towards an emotion (or avoid to reach one). 


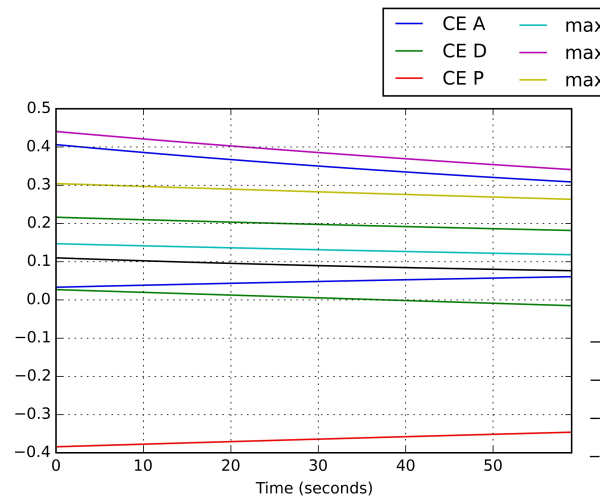

a
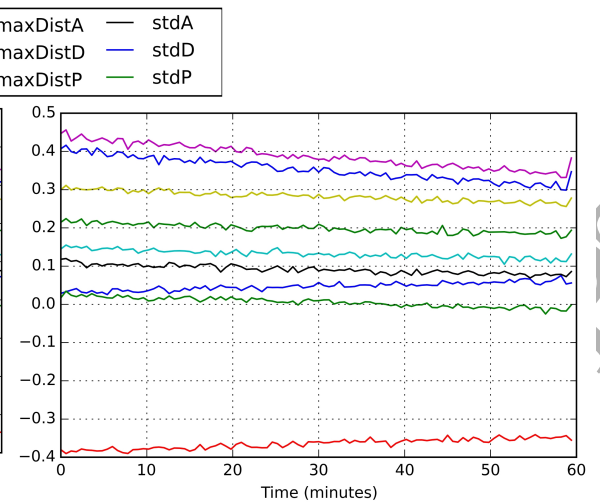

b

Figure 12: Group 2 - Experiment 2: Low Affinity levels and negative emotions.

\section{Conclusions and future work}

A new model for the calculation of dynamic emotions has been presented in this paper, showing a first approach for the emotional contagion and simulation of dynamic social emotions into a group of intelligent entities. The proposed model uses the personality of each entity and the affinity level between entities in order to calculate and represent the emotional dynamic of a group. The dynamic emotional model of a group of agents not only allows a global view of the emotional dynamic of the group, also can improve the decision making based on the attraction level between entities.

Specifically, the proposed model uses the dynamic Newton Law and universal gravitation law, to calculate the attraction level $\left(\overrightarrow{E A F}_{t}\left(a g_{i}\right)\right)$ and the new emotion of each agent $\left(\vec{E}_{t+1}\left(a g_{j}\right)=\vec{E}_{t}\left(a g_{j}\right)+\left(\vec{v}_{t}\left(a g_{i}\right) \cdot t\right)\right)$. These definitions allow to calculate the emotional attraction between entities or groups. Moreover, it is possible to obtain the resulting emotion of the attraction in a $(t+1)$, as well as the emotional propagation velocity $\left(\vec{v}_{t}\left(a g_{i}\right)\right)$ time. Considering these elements it is possible to know how is the emotional distribution among the agent group and to use this information to reason about future decisions.

The model evaluation was done through the use of a mobile robot application. 
Specifically, the proposed application consists of a NAO mobile robot that tries to interact with a group of people in a room. Results show that if the robot implements our proposed model, it is able to estimate the dynamic behaviour of people from an emotional point of view. Using these estimations, the robot may be able to enhance its decision-making process.

As future work, we want to introduce emotion recognition using physiological signals. Using this information we predict that it is possible of enhancing the emotion detection and improving the detection time of the human emotion.

\section{Acknowledgements}

This work is partially supported by the MINECO/FEDER TIN2015-65515-C4-1$\mathrm{R}$ and the FPI grant AP2013-01276 awarded to Jaime-Andres Rincon. This work is supported by COMPETE: POCI-01-0145-FEDER-007043 and FCT - Fundacao para a

Ciencia e Tecnologia within the projects UID/CEC/00319/2013 and Post-Doc scholarship SFRH/BPD/102696/2014 (A. Costa).

\section{References}

[1] W. James, What Is an Emotion?, Wilder Publications, 2007.

[2] J. W. Pennebaker, The Secret Life of Pronouns: What Our Words Say about Us, BLOOMSBURY, 2011.

[3] N.A. Fox, S. D. Calkins, The development of self-control of emotion: Intrinsic and extrinsic influences, Motivation and emotion 27 (1) (2003) 7-26.

[4] R. R. McCrae, O. P. John, An introduction to the five-factor model and its applications, Journal of personality (2) (1992) 175-215.

[5] A. Ortony, The Cognitive Structure of Emotions, Cambridge University Press, 1990.

[6] R. Plutchik, A general psychoevolutionary theory of emotion, Theories of emotion 1 (1980) 3-31. 
[7] A. Mehrabian, Analysis of affiliation-related traits in terms of the PAD temperament model, The Journal of Psychology 131 (1) (1997) 101-117. doi: $10.1080 / 00223989709603508$.

[8] C. Busso, Z. Deng, et al, Analysis of emotion recognition using facial expressions, speech and multimodal information, in: Proceedings of the 6th International Conference on Multimodal Interfaces, ICMI '04, ACM, New York, NY, USA, 2004, pp. 205-211. doi:10.1145/1027933.1027968.

[9] B. Schuller, G. Rigoll, M. Lang, Hidden markov model-based speech emotion recognition, in: Acoustics, Speech, and Signal Processing, 2003. Proceedings.(ICASSP'03). 2003 IEEE International Conference on, Vol. 2, IEEE, 2003, pp. II-1.

595 [10] D. O. Bos, EEG-based emotion recognition, The Influence of Visual and Auditory Stimuli (2006) 1-17.

[11] J. Saunier, H. Jones, in: Proc. of the 2014 international conference on Autonomous agents and multi-agent systems, pp. 645-652.

[12] T. Bosse, R. Duell, Z. A. Memon, J. Treur, C. N. Van Der Wal, A multi-agent model for emotion contagion spirals integrated within a supporting ambient agent model, in: Principles of Practice in Multi-Agent Systems, Springer, 2009, pp. 48-67.

[13] J. Rincon, A. Costa, P. Novais, V. Julian, C. Carrascosa, A dynamic emotional model for agent societies, in: Advances in Practical Applications of Scalable Multi-agent Systems. The PAAMS Collection, Springer, 2016, pp. 169-182.

[14] Â. Costa, J. C. Castillo, P. Novais, A. Fernández-Caballero, R. Simoes, Sensordriven agenda for intelligent home care of the elderly, Expert Systems with Applications 39 (15) (2012) 12192-12204. doi:10.1016/j .eswa. 2012. 04.058. 
[15] A. Costa, P. Novais, R. Simoes, A caregiver support platform within the scope of an ambient assisted living ecosystem., Sensors 14 (3) (2014) 5654-5676. doi : $10.3390 /$ s140305654.

[16] C. Adam, R. Canal, B. Gaudou, H. T. Vinh, P. Taillandier, others, Simulation of the Emotion Dynamics in a Group of Agents in an Evacuation Situation, in: Principles and Practice of Multi-Agent Systems, Springer, 2012, pp. 604-619.

[17] S. Banarjee, C. Grosan, A. Abraham, Emotional ant based modeling of crowd dynamics, in: Symbolic and Numeric Algorithms for Scientific Computing, 2005. SYNASC, IEEE, 2005, pp. 8-pp.

[18] G. I. Hawe, G. Coates, D. T. Wilson, R. S. Crouch, Agent-based simulation for large-scale emergency response: A survey of usage and implementation, ACM Computing Surveys (CSUR) 45 (1) (2012) 8.

[19] V. Lungu, Newtonian emotion system, in: Intelligent Distributed Computing VI, Springer, 2013, pp. 307-315.

[20] T. Bosse, M. Hoogendoorn, et al, Modelling collective decision making in groups and crowds: Integrating social contagion and interacting emotions, beliefs and intentions, JAAMAS 27 (1) (2013) 52-84. doi:10.1007/ s10458-012-9201-1.

[21] S. G. Barsade, The ripple effect: Emotional contagion and its influence on group behavior, Administrative Science Quarterly 47 (4) (2002) 644-675. doi: $10.2307 / 3094912$.

[22] J. Pripfl, T. Kortner, D. Batko-Klein, D. Hebesberger, M. Weninger, C. Gisinger, S. Frennert, H. Eftring, M. Antona, I. Adami, A. Weiss, M. Bajones, M. Vincze, Results of a real world trial with a mobile social service robot for older adults, in: 2016 11th ACM/IEEE International Conference on Human-Robot Interaction (HRI), Institute of Electrical and Electronics Engineers (IEEE), 2016. doi:10.1109/hri.2016.7451824. 
[23] D. Fischinger, P. Einramhof, K. Papoutsakis, W. Wohlkinger, P. Mayer, P. Panek, S. Hofmann, T. Koertner, A. Weiss, A. Argyros, M. Vincze, Hobbit, a care robot supporting independent living at home: First prototype and lessons learned, Robotics and Autonomous Systems 75 (2016) 60-78. doi:10.1016/j robot. 2014.09 .029 .

[24] J. Stuckler, M. Schwarz, S. Behnke, Mobile manipulation, tool use, and intuitive interaction for cognitive service robot cosero, Frontiers in Robotics and AI 3 (2016) 58. doi:10.3389/frobt. 2016.00058.

[25] J. Lunenburg, S. van den Dries, R. Appeldoorn, R. Wijnands, M. van de Molengraft, Tech united eindhoven @home 2016 team description paper, Tech. rep., Eindhoven University of Technology (2016).

[26] N. M. McDonald, D. S. Messinger, A. Acerbi, J. A. Lombo, J. J. Sanguineti, The development of empathy: How, when, and why, Moral Behavior and Free Will: A Neurobiological and Philosophical Aprroach (2011) 341-368.

[27] J. A. Rincon, V. Julian, C. Carrascosa, Social Emotional Model, in: Advances in Practical Applications of Agents, Multi-Agent Systems, and Sustainability: The PAAMS Collection, Springer, 2015, pp. 199-210.

[28] E. Niewiadomska-Szynkiewicz, A. Sikora, Progress in Automation, Robotics and Meaşuring Techniques, Advances in Intelligent Systems and Computing 351 (2015) 181-190. doi : 10 . 1007/978-3-319-15847-1.

[29] M. M. F. Donia, A. A. A. Youssif, A. Hashad, Spontaneous facial expression recognition based on histogram of oriented gradients descriptor, Computer and Information Science 7 (2014) 31-37.

[30]

A. Dhall, R. Goecke, J. Joshi, M. Wagner, T. Gedeon, Emotion recognition in the wild challenge (emotiw) challenge and workshop summary, in: Proceedings of the 15th ACM on International Conference on Multimodal Interaction, ICMI '13, ACM, New York, NY, USA, 2013, pp. 371-372. doi: 10.1145/2522848.2531749. 
[31] J. A. Russell, A circumplex model of affect, Journal of Personality and Social Psychology 39 (1980) 1161-1178.

[32] O. Langner, R. Dotsch, G. Bijlstra, D. Wigboldus, S. Hawk, van Knippenberg A., Presentation and validation of the radboud faces database. cognition and emotion, 24(8), 1377-1388.doi : 10.1080-02699930903485076.

[33] M. S. Unluturk, K. Oguz, C. Atay, Emotion recognition using neural networks, in: Proceedings of the 10th WSEAS International Conference on Neural Networks, NN'09, World Scientific and Engineering Academy and Society (WSEAS), Stevens Point, Wisconsin, USA, 2009, pp. 82-85.

[34] P. Michel, R. El Kaliouby, Real time facial expression recognition in video using support vector machines, in: Proceedings of the 5th International Conference on Multimodal Interfaces, ICMI '03, ACM, New York, NY, USA, 2003, pp. 258264. doi : $10.1145 / 958432.958479$.

[35] M. C. Melchers, M. Li, B. W. Haas, M. Reuter, L. Bischoff, C. Montag, Similar personality patterns are associated with empathy in four different countries, Frontiers in Psychology 7 (2016) 290. 


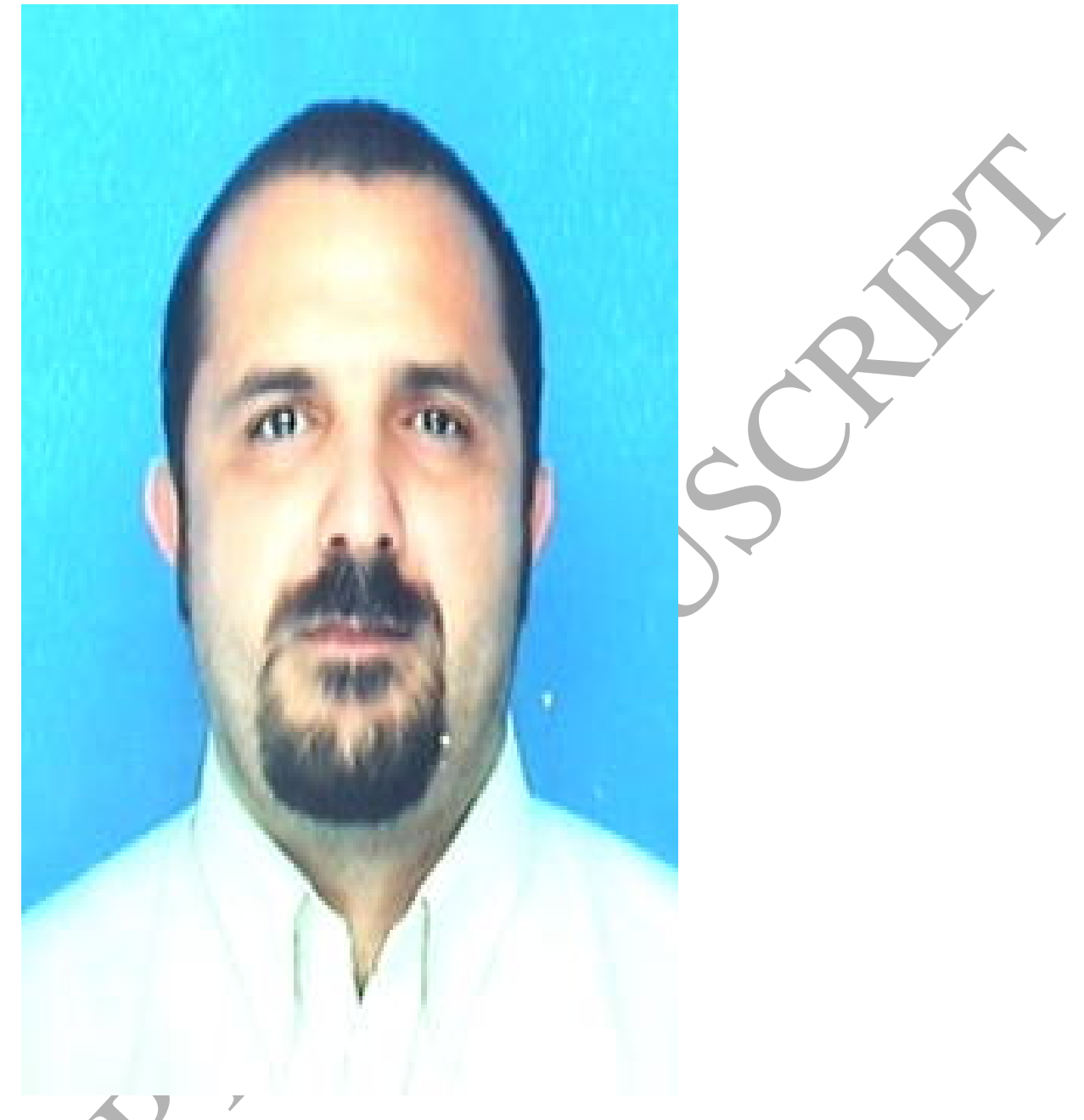

Jaime Andre Rincon was born in Buga (Valle), Colombia, in 1978. He received the B.E. degree in biomedical engineering from the University Manuela Beltran, Colombia, in 2008, and the MS degree in Artificial Intelligence from Universitat Politècnica de València. He is now a PhD student on Computer Sciences at the Universitat Politècnica de València. As a researcher his interest is in multi-agent systems, robotics and emotional agents. 


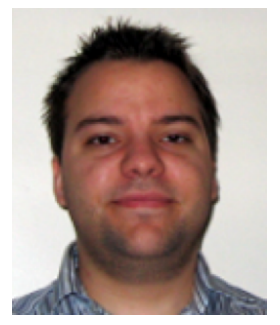

Ângelo Costa has received his European Doctorate title at the University of

690 Minho (Portugal) in 2013. He has also received his Master's title in 2009 by the University of Minho. Previously he worked as an Invited Assistant Professor at the Polytechnic of Porto, more specifically at the School of Management and Technology. As a researcher his interest is in multi-agent systems, ambient intelligence, mobile computing, machine learning, virtual organizations and privacy and data protection. He has participated in different research projects focused on Ambient Intelligence and Information Analysis both at a European and national level such as AAL4ALL, TIARAC. Additionally, he has been member of the organizing and scientific committee of several international symposiums such as PAAMS, ISAmI, PROVE, IDC, etc.

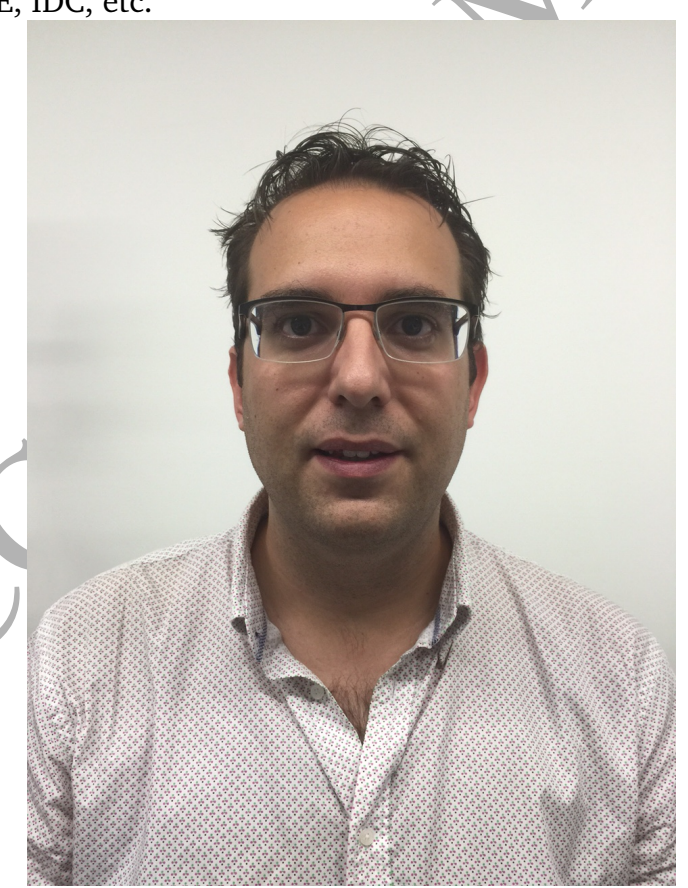




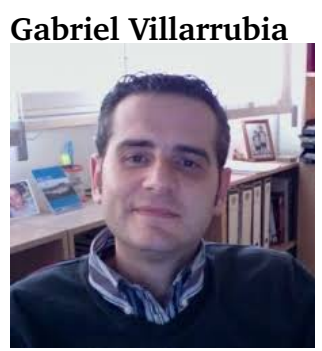

Vicente Julian holds a position of Associate Professor of Computer Science at the Universitat Politècnica de València (UPV) where he has taught since 1996. Vicente Julian is member of the GTI-IA research group, and Deputy Director of the Official Master in Artificial Intelligence, Pattern Recognition and Digital Imaging at the UPV. Four international projects, two international excellence networks, twenty one Spanish projects and four technology transfer projects have covered the research on Artificial Intelligence. He has more than 50 works published in journals with outstanding positions in the list of the Journal Citation Reports, or published in conference proceedings that have a system of external peer review and dissemination of knowledge comparable to journals indexed in relevant positions. Moreover, he has more than 130 contributions and a h-index of 22. Vicente Julian has supervised 8 PhD Thesis.

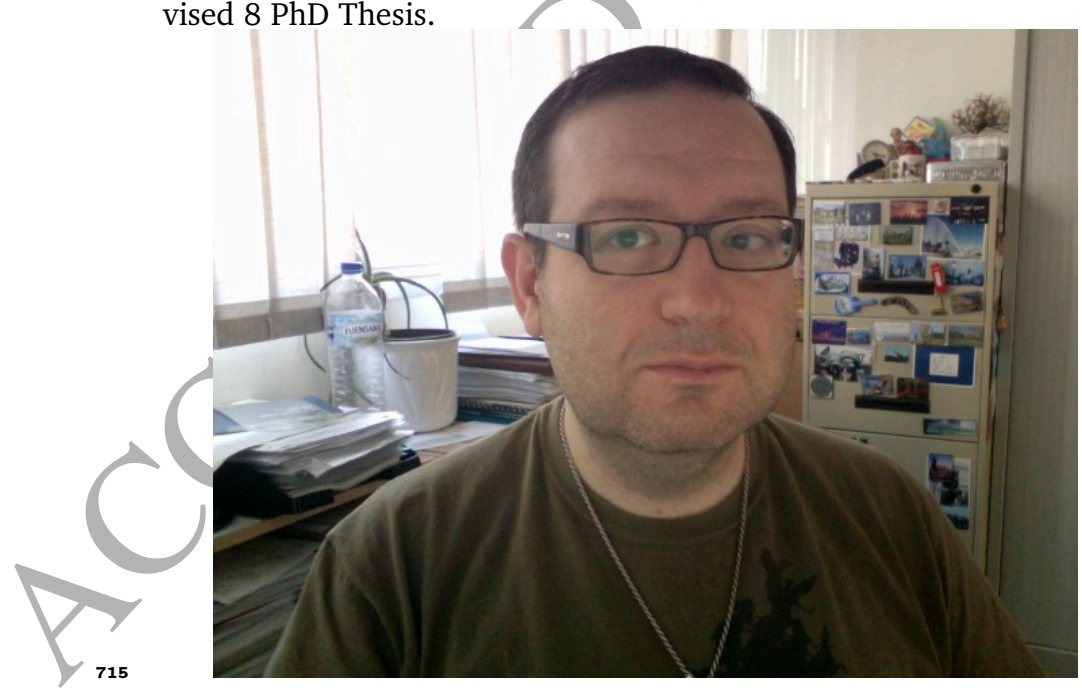

Carlos Carrascosa was born in Valencia (Spain) and received the M.S. degree in Computer Science from the Universidad Politécnica de Valencia (UPV) in 1995. He 
obtained his Ph.D. in the Departamento de Sistemas Informáticos y Computación at UPV and is currently a lecturer involved in teaching several AI-related subjects at

720 the UPV. His research interests include MAS, social emotions, consensus in MAS, Intelligent Virtual Environments, learning, serious games, information retrieval, and real-time systems. 